\title{
Pseudoharmonic oscillator in quantum mechanics with a minimal length
}

\author{
Djamil Bouaziz* \\ Laboratoire de Physique Théorique, Département de Physique, \\ Faculté des Sciences Exactes et Informatique, \\ Université de Jijel, BP 98, Ouled Aissa, 18000 Jijel, Algeria \\ Abdelmalek Boukhellout \\ Laboratoire de Physique de Rayonnement et Applications, \\ Département de Physique, Faculté des Sciences Exactes et Informatique, \\ Université de Jijel, BP 98, Ouled Aissa, 18000 Jijel, Algeria
}

(Date textdate; Received textdate; Revised textdate; Accepted textdate; Published textdate)

\begin{abstract}
The pseudoharmonic oscillator potential is studied in non relativistic quantum mechanics with a generalized uncertainty principle characterized by the existence of a minimal length scale, $(\Delta x)_{\min }=\hbar \sqrt{5 \beta}$. By using a perturbative approach, we derive an analytical expression of the energy spectrum in the first order of the minimal length parameter $\beta$. We investigate the effect of this fundamental length on the vibration-rotation energy levels of diatomic molecules through this potential function interaction. We explicitly show that the minimal length would have some physical importance in studying the spectra of diatomic molecules.
\end{abstract}

*Electronic address: djamilbouaziz@univ-jijel.dz

${ }^{\dagger}$ Electronic address: abdelmalek-boukhellout@univ-jijel.dz 


\section{INTRODUCTION}

Several studies in quatum gravity propose to modify the standard Heisenberg uncertainty principle to the form: $(\Delta X)(\Delta P) \geq \frac{\hbar}{2}\left(1+\beta(\Delta P)^{2}+\ldots\right)$, where $\beta$ is a small positive parameter [1 3]. This generalized uncertainty principle (GUP) includes a nonzero minimal uncertainty in position (minimal length), given by $(\Delta X)_{\min }=\hbar \sqrt{\beta}$. This minimal length is assumed to be on the order of the Planck length of $10^{-35} \mathrm{~m}$, which is a lower bound to all physical length scales [4-6]. The introduction of the GUP in quantum theory leads to fundamental consequences on the mathematical basis of quantum mechanics. One of the most important implications of this GUP is the deformation of the commutation relation

between position and momentum operators to the form: $[\widehat{X}, \widehat{P}]=i \hbar\left(1+\beta \widehat{P}^{2}\right)[7]$. The formalism, based on this deformed algebra, together with the new concepts it implies, has been discussed extensively in one and more dimensions by Kempf and co-workers in Refs. $[7-11]$.

In recent years, various topics were studied in this modified version of quantum mechanics, amongst other, we cite, the Schrödinger equation for, the harmonic oscillator potential [7, 12, 13], the hydrogen atom problem [12, 14-17], the singular inverse square potential [18, 19], the problem of a particle in a gravitational quantum well [20, 21]. In relativistic equations, the minimal length was introduced in the Dirac equation, with a constant magnetic field [22, 23], with vector and scalar linear potentials [24], for the hydrogen atom potential [25], and the Dirac oscillator [26, 27]. The effect of the GUP was also investigated in the context of the Klein-Gordon equation [22, 28, 29]. Finally, the Casimir effect [30, 31], and the black body radiation [32] have been studied within this modified formalism of quantum mechanics. For a large number of references in connection with this subject, see, Ref. [33]. For the sake of completness, let us mention that there is also another form of the GUP, recently proposed in the literature, which incorporates a minimal length and a maximal momentum [34, 35]. Much attention is equally given to the study of the implications of such GUP on physical problems, see, for instance, Refs. [36 39 39].

Only a few problems have been solved exactly in the formalism of quantum mechanics with a minimal length, such as the Schrödinger equation for the harmonic oscillator [13] and for the singular inverse square potential [18, 19]. In the hydrogen atom problem, for instance, the effect of the minimal length is assumed to be too small and studied perturbatively in 
coordinate space [12, 14,16$]$.

It is important to note that any theory based on the GUP is supposed to account for quantum gravitational effects, which arise at scales of the order of the Planck length [40]. However, it has been argued that, in relativistic or nonrelativistic quantum mechanics, this elementary length incorporated in the GUP may be viewed as an intrinsic scale characterizing the system under study [8, 18]. Consequently, the formalism based on these deformed commutation relations may provide a new model for an effective description of complex systems such as quasiparticles, nuclei, and molecules [8].

The purpose of this paper is to study another interaction, in quantum mechanics with a GUP, namely, the molecular pseudoharmonic oscillator $(\mathrm{PHO}), V(r)=D_{e}\left(\frac{r}{r_{e}}-\frac{r_{e}}{r}\right)^{2}$, where $D_{e}$ is the dissociation energy and $r_{e}$ is the equilibrium internuclear distance [41]. The PHO is one of the most important molecular potentials; it is especially relevant in studying diatomic molecules, and it is of great importance in chemical physics, molecular physics and other fields of physics [42 47]. The main feature of the Schrödinger equation with this potential function is that it allows its exact solution for arbitrary rotational quantum number $\ell$. This is an advantage compared to other molecular potentials such as the well-known Morse potential [48], which can solved exactly only for non-rotating diatomic molecules.

The objective of this work is twofold, first to compute perturbatively the energy spectrum of the PHO potential in the context of the deformed Schrödinger equation with a minimal length, and second, to investigate the effect of the deformation parameter on the vibrational and rotational energy levels of diatomic molecules. Of course, in such application, quantum gravitational effects are extremely small, and the the minimal length would not be on the order of the Planck scale but rather it might be associated to a dimension of the molecule.

The rest of this paper is organized as follows. In Sec. II, we present the basic equations of quantum mechanics with a GUP. Sec. III is devoted to the study of the PHO in quantum mechanics with a GUP, and the effect of the minimal length included in the formalism, on the vibration-rotation energy levels of diatomic molecules. In the last section, we summarize our results and conclusions. 


\section{BASIC EQUATIONS OF QUANTUM MECHANICS WITH A GUP}

We study the PHO potential in deformed quantum mechanics based on the following 3-dimensional modified Heisenberg algebra [7, 8]]:

$$
\begin{aligned}
{\left[\widehat{X}_{i}, \widehat{P}_{j}\right] } & =i \hbar\left[\left(1+\beta \widehat{P}^{2}\right) \delta_{i j}+\beta^{\prime} \widehat{P}_{i} \widehat{P}_{j}\right] \\
{\left[\widehat{P}_{i}, \widehat{P}_{j}\right] } & =0 \\
{\left[\widehat{X}_{i}, \widehat{X}_{j}\right] } & =i \hbar \frac{2 \beta-\beta^{\prime}+\beta\left(2 \beta+\beta^{\prime}\right) \widehat{P}^{2}}{1+\beta \widehat{P}^{2}}\left(\widehat{P}_{i} \widehat{X}_{j}-\widehat{X}_{i} \widehat{P}_{j}\right) .
\end{aligned}
$$

These commutators imply the following generalized uncertainty principle (GUP):

$$
\left(\Delta X_{i}\right)\left(\Delta P_{i}\right) \geq \frac{\hbar}{2}\left(1+\beta \sum_{j=1}^{3}\left[\left(\Delta P_{j}\right)^{2}+\left\langle\widehat{P}_{j}\right\rangle^{2}\right]+\beta^{\prime}\left[\left(\Delta P_{i}\right)^{2}+\left\langle\widehat{P}_{i}\right\rangle^{2}\right]\right)
$$

which leads to a lower bound of $\Delta X_{i}$, the so-called minimal length, given by [8, 12]

$$
\left(\Delta X_{i}\right)_{\min }=\hbar \sqrt{3 \beta+\beta^{\prime}}, \quad \forall i
$$

A fundamental consequence of the generalized uncertainty priciple is the loss of localization in coordinate space due to the presence of a nonzero minimal uncertainty in position measurements, so that, momentum space would be more convenient for solving any eigenvalue problem.

The most used momentum representation, satisfying Eqs. (1), is that given for the first time in Ref. [8]

$$
\widehat{X}_{i}=i \hbar\left(\left(1+\beta p^{2}\right) \frac{\partial}{\partial p_{i}}+\beta^{\prime} p_{i} p_{j} \frac{\partial}{\partial p_{j}}+\gamma p_{i}\right), \widehat{P}_{i}=p_{i}
$$

where $\gamma$ is related to $\beta$ and $\beta^{\prime}$.

The problem with this representation is that the solution to the deformed Schrödinger equation is not often simple; only a few problems have been solved exactly in momentum space with a minimal length. For instance, the hydrogen atom potential, with representation (4), can be solved only for the $s$-waves, in the case $\beta^{\prime}=2 \beta$ and in the first order of $\beta$ [17].

The other important representation used in the literature is the following coordinate space representation [12]: 


$$
\widehat{X}_{i}=\widehat{x}_{i}, \quad \widehat{P}_{i}=\widehat{p}_{i}\left(1+\beta \widehat{p}^{2}\right),
$$

which is valid in the particular case $\beta^{\prime}=2 \beta$, up to the first order of $\beta$. The operators $\widehat{x}_{i}$ and $\widehat{p}_{i}$ satisfy the standard commutation relations of ordinary quantum mechanics.

The main advantage of representation (5) is that it allows to apply the perturbation theory in order to study the Schrödinger equation with a given interaction.

In the following section we will study the Shrödinger equation for the PHO molecular potential using representation (5), we will be interested in particular to the effect of the minimal length on the energy spectrum.

\section{PHO POTENTIAL IN QUANTUM MECHANICS WITH A GUP}

While several potentials were studied in quantum mechanics with a GUP, here we consider the PHO molecular potential, which has the form

$$
V(r)=D_{e}\left(\frac{r}{r_{e}}-\frac{r_{e}}{r}\right)^{2},
$$

where $D_{e}$ is the dissociation energy and $r_{e}$ is the equilibrium internuclear distance of a given diatomic molecule [41].

As is clear, the potential (6) contains both the harmonic and the inverse square interactions, for which the deformed Schrödinger equation has been solved exactly with the momentum representation (44), see Refs. [13, 18]. However, the incorporation of the PHO

interaction into Schrödinger equation in momentum space leads to a differential equation of fourth order, for which the analytical solution is not known.

Given the importance of such potential, especially in molecular physics, we investigate here its deformed Schrödinger equation in coordinate space by using the representation (5).

We proceed then by writing the Schrödinger equation, for a particule with reduced mass $\mu$ interacting with the $\mathrm{PHO}$ potential in position representation as follows:

$$
\left(\frac{\widehat{P}^{2}}{2 \mu}+V(r)\right) \psi(\vec{r})=E \psi(\vec{r}) .
$$

Using representation (15) in Eq. (77), and neglecting terms of order $\beta^{2}$, we obtain the 
following deformed Schrödinger equation:

$$
\left(\frac{\widehat{p}^{2}}{2 \mu}+V(r)+\frac{\beta}{\mu} \widehat{p}^{4}\right) \psi(\vec{r})=E \psi(\vec{r}) .
$$

In the limit $\beta=0$, Eq. (8) reduces to the ordinary Schrödinger equation. Its solution for this potential function can be found in the standard textbooks of quantum mechanics, see, for instance, Ref. [49]. For the bound state solutions, the energy eigenvalues and the corresponding normalized eigenfunctions are [41, 49]:

$$
\begin{gathered}
E_{n \ell}^{0}=-2 D_{e}\left(1-\frac{1}{\gamma}(2 n+1+\lambda)\right), \quad n=0,1,2, \ldots \\
\psi_{n \ell m}^{0}(r, \theta, \varphi)=N_{n \ell} r^{-\frac{1}{2}+\lambda} \exp \left(-\frac{\alpha}{2} r^{2}\right){ }_{1} F_{1}\left(-n, 1+\lambda ; \alpha r^{2}\right) Y_{\ell m}(\theta, \varphi),
\end{gathered}
$$

where $N_{n \ell}$ is a normalization constant given by

$$
N_{n \ell}=\frac{\alpha^{\frac{1}{2}+\frac{\lambda}{2}}}{\Gamma(\lambda+1)} \sqrt{\frac{2 \Gamma(\lambda+n+1)}{n !}},
$$

${ }_{1} F_{1}$ is a confluent hypergeometric function, $Y_{\ell m}$ are the spherical harmonics, $n$ and $\ell$ are, respectively, the radial (vibrational) and orbiatal (rotational) quantum numbers. We have used the notations

$$
\gamma=\sqrt{\frac{2 \mu D_{e} r_{e}^{2}}{\hbar^{2}}}=\frac{4 D_{e}}{\hbar \omega}, \lambda=\sqrt{\gamma^{2}+\left(\ell+\frac{1}{2}\right)^{2}}, \quad \alpha=\frac{\gamma}{r_{e}^{2}}, \quad \omega=\frac{2}{r_{e}} \sqrt{\frac{2 D_{e}}{\mu}},
$$

where $\omega$ represents the classical frequency for small harmonic vibrations.

We return now to Eq. (8) . It is clearly seen that the effect of the minimal length is described by the presence of the perturbation term $\frac{\beta}{\mu} \widehat{p}^{4}$ in the ordinary Schrödinger equation. Thus, one can use the perturbation theory to compute the correction to the energy levels in the first order in $\beta$. We can then write

$$
E_{n \ell}=E_{n \ell}^{0}+\Delta E_{n \ell}
$$

where $E_{n \ell}^{0}$ are the unperturbed levels corresponding to the eigenfunctions $\psi_{n \ell m}^{0}(r)$, solutions to the ordinary Schrödinger equation, and $\Delta E_{n \ell}$ is the correction caused by the minimal length. It is given by

$$
\Delta E_{n \ell}=\frac{\beta}{\mu}\left\langle\psi_{n \ell m}^{0}\left|p^{4}\right| \psi_{n^{\prime} \ell^{\prime} m^{\prime}}^{0}\right\rangle \equiv \frac{\beta}{\mu}\left\langle n \ell m\left|p^{4}\right| n^{\prime} \ell^{\prime} m^{\prime}\right\rangle .
$$


It has been shown in Ref. [12] that, for central interactions, $\Delta E_{n \ell}$ can be expressed as follow:

$$
\Delta E_{n \ell}=4 \beta \mu\left[\left(E_{n \ell}^{0}\right)^{2}-2 E_{n \ell}^{0}\langle n \ell m|V(r)| n \ell m\rangle+\left\langle n \ell m\left|V^{2}(r)\right| n \ell m\right\rangle\right] .
$$

For the sake of later comparison with the harmonic oscillator, we write the PHO potential (6]) in the general form

$$
V(r)=a r^{2}+\frac{b}{r^{2}}+c
$$

where the parameters $a, b$ and $c$ are related to the dissociation energy $D_{e}$ and to the equilibrium internuclear distance $r_{e}$ by

$$
a=D_{e} / r_{e}^{2}, \quad b=D_{e} r_{e}^{2}, \quad c=-2 D_{e}
$$

By using formula (11) with the PHO potential (12), we obtain

$$
\begin{aligned}
& \Delta E_{n \ell}=4 \mu \beta\left(\left(E_{n \ell}^{0}\right)^{2}+2 a b+c^{2}-2 c E_{n \ell}^{0}+\left(2 a c-2 a E_{n \ell}^{0}\right)\left\langle n \ell m\left|r^{2}\right| n \ell m\right\rangle\right. \\
& \left.+a^{2}\left\langle n \ell m\left|r^{4}\right| n \ell m\right\rangle+\left(2 b c-2 b E_{n \ell}^{0}\right)\left\langle n \ell m\left|\frac{1}{r^{2}}\right| n \ell m\right\rangle+b^{2}\left\langle n \ell m\left|\frac{1}{r^{4}}\right| n \ell m\right\rangle\right) .
\end{aligned}
$$

To compute the minimal length correction, one has then to evaluate the matrix elements

$$
\left\langle n \ell m\left|r^{q}\right| n \ell m\right\rangle=N_{n, \ell}^{2} \int_{0}^{\infty} r^{q+1+2 \lambda} \exp \left(-\alpha r^{2}\right)\left[{ }_{1} F_{1}\left(-n, 1+\lambda ; \alpha r^{2}\right)\right]^{2} d r,
$$

where $q=-4,-2,2,4$.

After evaluating the above integrals, we have obtained the following results:

$$
\begin{aligned}
\left\langle n \ell m\left|r^{2}\right| n \ell m\right\rangle & =\frac{\lambda+2 n+1}{\alpha}, \\
\left\langle n \ell m\left|r^{4}\right| n \ell m\right\rangle & =\frac{(\lambda+1)(\lambda+2)+6 n(\lambda+n+1)}{\alpha^{2}} \\
\left\langle n \ell m\left|\frac{1}{r^{2}}\right| n \ell m\right\rangle & =\frac{\alpha}{\lambda}, \\
\left\langle n \ell m\left|\frac{1}{r^{4}}\right| n \ell m\right\rangle & =\alpha^{2} \frac{\lambda+2 n+1}{\lambda\left(\lambda^{2}-1\right)} .
\end{aligned}
$$

By inserting these results in Eq. (13), we arrive at the expression

$$
\begin{gathered}
\Delta E_{n \ell}=4 \mu \beta\left(\left(\left(E_{n \ell}^{0}\right)^{2}+2 a b+c^{2}-2 c E_{n \ell}^{0}\right)+\sqrt{\frac{a \hbar^{2}}{2 \mu}}\left(2 c-2 E_{n \ell}^{0}\right)(\lambda+2 n+1)+\right. \\
\left.a \frac{\hbar^{2}}{2 \mu}((\lambda+1)(\lambda+2)+6 n(\lambda+n+1))+\sqrt{\frac{2 \mu}{\hbar^{2}} a}\left(2 b c-2 b E_{n \ell}^{0}\right) \frac{1}{\lambda}+\frac{2 \mu}{\hbar^{2}} a b^{2} \frac{\lambda+2 n+1}{\lambda\left(\lambda^{2}-1\right)}\right) .
\end{gathered}
$$


Equation (14) generalizes the formula given in Ref. [12] for the harmonic oscillator potential, which is obtained when $b=c=0$. In this case one has

$$
\Delta E_{n \ell}^{h o}=4 \beta \mu a \frac{\hbar^{2}}{2 \mu}\left(\left(\ell+\frac{3}{2}\right)\left(\ell+\frac{5}{2}\right)+6 n\left(\ell+n+\frac{3}{2}\right)\right)
$$

which is exactly the correction derived in Ref. [12].

From Eqs.(9) and (14), the complete energy spectrum of the PHO in the presence of a minimal length can be written, in terms of the molecular parameters $r_{e}$ and $D_{e}$, as folow:

$$
\begin{gathered}
E_{n \ell}=E_{n \ell}^{0}+4 \mu \beta\left(\left(E_{n \ell}^{0}\right)^{2}+4 D_{e} E_{n \ell}^{0}+6 D_{e}^{2}-\left(4 D_{e}^{2}+2 D_{e} E_{n \ell}^{0}\right) \frac{\lambda+2 n+1}{\gamma}+\right. \\
\left.D_{e}^{2} \frac{\lambda^{2}+(6 n+3) \lambda+6 n(n+1)+2}{\gamma^{2}}-\gamma \frac{2 D_{e}\left(2 D_{e}+E_{n \ell}^{0}\right)}{\lambda}+D_{e}^{2} \gamma^{2} \frac{\lambda+2 n+1}{\lambda\left(\lambda^{2}-1\right)}\right)
\end{gathered}
$$

where, $n=0,1,2, \ldots$, and $\lambda=\sqrt{\gamma^{2}+\left(\ell+\frac{1}{2}\right)^{2}}$.

This formula is the main result of this work. It allows us to investigate the effect of the GUP on the vibration-rotation energy levels of a given diatomic molecule with the PHO interaction.

In fact, we may expand $E_{n, \ell}$ into powers of $1 / \gamma$ since the parameter $\gamma$ is so large $(\gamma \gg 1)$ for most molecules [48]. We obtain after some calculations and simplifications the expression

$$
\begin{aligned}
E_{n \ell} & =4 D_{e}\left(\left(n+\frac{1}{2}\right) \frac{1}{\gamma}+\frac{1}{4}\left(\ell+\frac{1}{2}\right)^{2} \frac{1}{\gamma^{2}}\right) \\
& +4 \mu \beta D_{e}^{2}\left\{[6 n(n+1)+3] \frac{1}{\gamma^{2}}+\left(n+\frac{1}{2}\right)\left[4\left(\ell+\frac{1}{2}\right)^{2}+2\right] \frac{1}{\gamma^{3}}\right\}+O\left(1 / \gamma^{4}\right),
\end{aligned}
$$

which can be approximated for a rotating diatomic molecules as follow:

$$
\begin{aligned}
E_{n \ell} & \approx 4 D_{e}\left(n+\frac{1}{2}\right) \frac{1}{\gamma}+D_{e}\left(\ell+\frac{1}{2}\right)^{2} \frac{1}{\gamma^{2}} \\
& +\left(6 \mu \beta D_{e}^{2}\right) \frac{1}{\gamma^{2}}+24 \mu \beta D_{e}^{2}\left(n+\frac{1}{2}\right)^{2} \frac{1}{\gamma^{2}}+16 \mu \beta D_{e}^{2}\left(n+\frac{1}{2}\right)\left(\ell+\frac{1}{2}\right)^{2} \frac{1}{\gamma^{3}}+O\left(\frac{1}{\gamma^{4}}\right) .
\end{aligned}
$$

This formula shows the effect of the deformed Heisenberg algebra on the energy levels of a diatomic molecule interacting with the PHO potential. We can observe that the minimal length correction carries new terms in the ro-vibrational energy spectrum, which do not 
exist in the undeformed case, such as the anharmonic vibrations and the coupling vibrationrotation. In fact, the third term in Eq. (17) can be viewed as a correction caused by the minimal length to the dissociation energy of the molecule. The fourth term is a correction that accounts for the anharmonicity of vibrations. Finally, the last term is simply the effect of the minimal length on the coupling vibration-rotation. However, it is easily seen, that the effect of the minimal length on the harmonic vibrational energy levels is too small; the correction of this energy is proportional to $1 / \gamma^{3}$, and is neglected compared to the first term in Eq. (17). This result seems to be interesting as long as the introduction of this elementary length brings all empirical terms of diatomic molecules energy spectrum. Therefore, the PHO potential becomes a more realistic model in this formalism of quantum mechanics with a minimal length; its spectrum is now similar to those of the well known Kratzer and Morse potentials [48].

Furthermore, Formula (16) can be viewed as an energy spectrum of a three-parameter potential function, i.e., $D_{e}, r_{e}$, and $\beta$. In this sense, the procedure of fitting used in the case of three-parameter potentials, such as Morse function, can be followed for adjusting the parameters of the "deformed PHO" with the spectroscopic data of diatomic molecules. This viewpoint would be important because one can compute the values of $\beta$ for any molecule, and estimate an order of magnitude of the minimal length in molecular physics. For this, it would be interesting to compute the energy spectrum in the general case $\left(\beta^{\prime} \neq 2 \beta\right)$ of the deformed Heisenberg algebra (1). This is because, in the modified Schrödinger equation (8), studied here, the perturbation term $(\beta / \mu) \widehat{p}^{4}$ can arise from many different sources, such as the relativistic corrections, and hence it can describe not only the minimal length. However, in the case $\left(\beta^{\prime} \neq 2 \beta\right)$, the Schrödinger equation takes a complicated specific form, which clearly describe the minimal length corrections. One can then, without any ambiguity, conclude about the significance, and the order of the minimal length in diatomic molecules physics.

\section{SUMMARY}

We studied the PHO potential in quantum mechanics with a GUP, which includes a minimal length. By using a perturbative approach, the correction to the energy spectrum of the PHO potential due to the existence of this fundamental length is obtained analytically 
in coordinate space. The problem of vibration-rotation of diatomic molecules is investigated in this modified version of quantum mechanics by means of this potential model. The effect

of the minimal length on the ro-vibrational energy levels manifests by the appearance of three kinds of corrections in the energy spectrum: the first modifies the dissociation energy, the second concerns the anharmonic vibrations, and the last correction affects the coupling vibration-rotation. This result shows that the introduction of the minimal length improves this molecular potential model since its energy spectrum includes now all the necessary energy terms to describe the experimental vibration-rotation energy levels of diatomic molecules. Therefore, It would be important to extend this study to the general case of the deformed Heisenberg algebra (arbitrary values of $\beta$ and $\beta^{\prime}$ ), and thereafter one can investigate quantitatively the effect of the minimal length on the vibration-rotation of diatomic molecules. This work is in progress and will be published else where.

\section{Acknowledgments}

This work was supported by the Algerian Ministry of Higher Education and Scientific Research under the PNR Project No. 8/u18/4327 and the CNEPRU Project No. D017201600026. We thank the referee for a pertinent remark that led us to clarify the conclusion of this study.

[1] L. J. Garay, J. Mod. Phys. A 10, 145 (1995).

[2] D. Amati, M. Ciafaloni and G. Veneziano, Phys. Lett. B 216, 41 (1989).

[3] M. Magiore, Phys. Lett. B 319, 83 (1993).

[4] T. Padmanabhan, Gen. Rel. Grav 17, 215 (1985).

[5] M. T. Jaekel and S. Reynaud, Phys. Lett. A 185, 143 (1994).

[6] X. CALMET, Mod. Phys. Lett. A, 22, 2027 (2007).

[7] A. Kempf, G. Mangano, and R. B. Mann, Phys. Rev. D 52, 1108 (1995).

[8] A. Kempf, J. Phys. A. 30, 2093 (1997).

[9] A. Kempf, J. Math. Phys. 35, 4483 (1994).

[10] A. Kempf, J. Math. Phys. 38, 1347 (1997). 
[11] A. Kempf and G. Mangano, Phys. Rev. D 55 , 7909 (1997).

[12] F. Brau, J. Phys. A 32, 7691 (1999).

[13] L. N. Chang, D. Minic, N. Okamura, and T. Takeuchi, Phys. Rev. D 65, 125027 (2002).

[14] S. Benczik, L. N. Chang, D. Minic, and T. Takeuchi, Phys. Rev. A 72, 012104 (2005).

[15] M. M. Stetsko and V. M. Tkachuk, Phys. Rev. A 74, 012101 (2006).

[16] M. M. Stetsko, Phys. Rev. A 74, 062105 (2006).

[17] D. Bouaziz and N. Ferkous, Phys. Rev. A. 82, 022105 (2010).

[18] D. Bouaziz and M. Bawin, Phys. Rev. A. 76, 032112 (2007).

[19] D. Bouaziz and M. Bawin, Phys. Rev. A. 78, 032110 (2008).

[20] F. Brau and F. Buisseret, Phys. Rev. D 74, 036002 (2006).

[21] P. Pedram, K. Nozari, and S. H. Taheri, J. High. Energy. Phys. 1103, 093 (2011).

[22] M. Merad, F. Zeroual, and M. Falek, Mod. Phys. Lett. A 27, 1250080 (2012).

[23] L. Menculini, O. Panella, and P. Roy, Phys. Rev. D 87, 065017 (2013).

[24] Y. Chargui, A. Trabelsi, and L. Chetouani, Phys. Lett. A. 374, 531 (2010).

[25] T. L. Antonacci Oakes, R. O. Francisco, J. C. Fabris, J. A. Nogueira, EPJC. 73, 2495 (2013).

[26] C. Quesne and V. M. Tkachuk, J. Phys. A : Math. Gen. 38, 1747 (2005).

[27] Kh. Nouicer, J. Phys. A: Math. Gen. 39, 5125 (2006).

[28] T. K. Jana, and P. Roy, Phys. Lett A. 373, 1239 (2009).

[29] S. K. Moayedi, M. R. Setare, and H. Moayeri, Int. J. Theor. Phys. 49, 2080 (2010).

[30] U. Harbach and S. Hossenfelder, Phys. Lett. B 632, 379 (2006).

[31] O. Panella, Phys. Rev. D. 76, 045012 (2007).

[32] D. Mania, and M. Maziashvili, Phys. Lett. B 705521 (2011).

[33] S. Hossenfelder, Living Rev. Relativity. 16, 2 (2013).

[34] A. F. Ali , S. Das, and E. C. Vagenas, Phys. Lett. B. 678, 497 (2009)

[35] Das S, E. C. Vagenas, and A. F. Ali, Phys. Lett. B 690, 407 (2010).

[36] K. Nozari and A. Etemadi, Phys. Rev. D 85, 104029 (2012).

[37] P. Pedram, Phys. Lett. B. 714, 317 (2012).

[38] C.L. Ching, and R.R. Parwani, Mod. Phys. Lett. A 28, 1350061 (2013).

[39] C.L. Ching and W.K. Ng, Mod. Phys. Lett. A 29, 1450080 (2014).

[40] M. Sprenger, P. Nicolini, and M. Bleicher, Eur. J. Phys. 33, 853 (2012).

[41] S. Ikhdair, R. Sever, J. Mol. Struct.: Theochem. 806, 155 (2007). 
[42] K. J. Oyewumi, K. D. Sen, J. Math Chem 50, 1039 (2012).

[43] S. Erkoc and R. Sever, Phys. Rev. 37, 2687 (1988).

[44] Maitland, G., et al.: Intermolecular Forces. Oxford University Press, Oxford (1987).

[45] Shigeru, A.: J. Math. Chem. 41, 3 (2007).

[46] Chakraborty, T.: Commun. Cond. Mat. Phys. 16, 35 (1992).

[47] Ashoori, R.: Nature 379, 413 (1996).

[48] S. Flügge, Practical quantum mechanics, (Springer-Verlag, 1994), pp. 178-189.

[49] L. Landau and E. M. Lifshitz, Quantum Mechanics, Vol. 3 (Pergamon Press, London, 1958), pp. $127-128$. 